\title{
A deletion in nephronophthisis 4 (NPHP4) is associated with recessive cone-rod dystrophy in standard wire-haired dachshund
}

\author{
Anne Caroline Wiik, ${ }^{1}$ Claire Wade, ${ }^{2,3}$ Tara Biagi, ${ }^{2}$ Ernst-Otto Ropstad, ${ }^{4}$ Ellen Bjerkås, ${ }^{4}$ \\ Kerstin Lindblad-Toh, ${ }^{2,5,6}$ and Frode Lingaas ${ }^{1,6}$ \\ ${ }^{1}$ Department of Basic Sciences and Aquatic Medicine, Division of Genetics, Norwegian School of Veterinary Science, \\ 0033 Oslo, Norway; ${ }^{2}$ Broad Institute of Harvard and MIT, Cambridge, Massachusetts 02141, USA; ${ }^{3}$ Center for Human Genetic \\ Research, Massachusetts General Hospital, Boston, Massachusetts 02114, USA; ${ }^{4}$ Department of Companion Animal Clinical \\ Sciences, Norwegian School of Veterinary Science, 0033 Oslo, Norway; ${ }^{5}$ Department of Medical Biochemistry and Microbiology, \\ Uppsala University, 75124 Uppsala, Sweden
}

\begin{abstract}
Cone-rod dystrophy is a retinal degenerative disorder occurring naturally in man and dog. Here we identify a novel gene for early-onset cone-rod dystrophy in the wire-haired dachshund. For the first time, we use genome-wide association-based Sibling Transmission Disequilibrium Test (sibTDT) analysis of only 13 discordant sib-pairs to identify a single significantly associated $6.5-\mathrm{Mb}$ region $\left(P_{\text {rawTDT }}=4.8 \times 10^{-5}, P_{\text {genomeTDT }}=6 \times 10^{-4}\right)$ on canine chromosome 5, containing more than 70 genes. Segregation studies using microsatellites in the candidate region including additional meiosis supported the sibTDT analysis but could not further reduce the area. Candidate gene resequencing identified a 180-bp deletion in exon/intron 5 of NPHP4 (nephronophthisis 4, also known as nephroretinin). RT-PCR analysis of NPHP4 in cases and controls showed exon skipping of exon 5, resulting in a truncated protein that retains the binding domain interacting with nephronophthisis 1 (also known as nephrocystin-1) in the kidney but lacks the domain interacting with RPGRIPI in retina. We suggest that this deletion in the canine NPHP4 gene is the cause of cone-rod dystrophy in the standard wire-haired dachshund. In humans, mutations in NPHP4 have been associated with simultaneous eye and kidney disease. Here we describe the first naturally occurring mutation in NPHP4 without additional kidney disease. Further studies will permit elucidation of the complex molecular mechanism of this retinopathy and the development of potential therapies.
\end{abstract}

[Supplemental material is available online at www.genome.org. The sequence data from this study have been submitted to GenBank under accession nos. EU707775-EU707790.]

The cone-rod dystrophies (crds) are a heterogeneous group of disorders occurring naturally in man and dog and are characterized by a predominant loss of cone function, with relative preservation of rod function (Udar et al. 2003; Michaelides et al. 2005). A few genes have been associated with autosomal recessively inherited crd in man, including $A B C A 4$ (Allikmets et al. 1997a,b) and RPGRIP1 (Hameed et al. 2003) as well as the loci CORD8 (Khaliq et al. 2000; Ismail et al. 2006) and CORD9 (Danciger et al. 2001). This number of identified loci associated with crd is low compared with the large number of $\sim 130$ genes identified for eye diseases in general (RetNet).

Cone-rod dystrophy is classified as a retinal ciliopathy, together with many other diseases including retinitis pigmentosa, macular degeneration, cone dystrophy, Leber congenital amaurosis (LCA), and Joubert syndrome (JBTS) (Adams et al. 2007). Several groups of genes have been implicated in these studies, including retinitis pigmentosa-1 (RP1), retinitis pigmentosa GTPase regulator $(R P G R)$, retinitis pigmentosa GTPase regulator interacting protein (RPGRIP1), as well as the Usher, Bardet-Biedl, and nephronophthisis genes (Adams et al. 2007). These genes are expressed in the outer segment of the photoreceptors, which is a

\footnotetext{
${ }^{6}$ Corresponding authors.
}

E-mail kersli@broad.mit.edu; fax (617) 258-0903.

E-mail Frode.Lingaas@veths.no; fax +47-22597310.

Article published online before print. Article and publication date are at http:// www.genome.org/cgi/doi/10.1101/gr.074302.107. specialized and modified cilium, where they have a structural role and are crucial for transport function. They also are involved in protein-protein interaction with transport-like proteins (Adams et al. 2007).

Nephronophthisis (NPHP) comprises a group of autosomal recessive cystic kidney diseases, which constitute the most frequent genetic cause for end-stage renal failure in children and young adults. Six genes, NPHP1, INVS (formerly known as NPHP2), NPHP3, NPHP4, IQCB1 (NPHP5), CEP290 (NPHP6), have been identified as mutated in human NPHP. In JBTS, retinal degeneration is associated with mental retardation, cerebellar vermis aplasia/hypoplasia, and NPHP (Parisi et al. 2004). Mutations in CEP290 and RPGRIP1L, homolog of RPGRIP1 and nephronophthisis 4 (NPHP4, also known as nephroretinin and nephrocystin-4) interactor, are involved in JBTS (Valente et al. 2006; Arts et al. 2007). In Senior-Loken syndrome (SLSN), NPHP are associated with retinitis pigmentosa, tapetoretinal degeneration, or retinal dysplasia (Hildebrandt et al. 2007). SLSN is present in about $10 \%$ of people with mutations in NPHP1, INVS, NPHP3, or NPHP4 (Caridi et al. 1998; Otto et al. 2002; Olbrich et al. 2003; O'Toole et al. 2007) and $>90 \%$ of people with mutations in IQCB1 or CEP290 (Otto et al. 2005; Sayer et al. 2006). RPGRIP1 and NPHP4 interact strongly in vitro and in vivo, and they colocalize in the retina (Roepman et al. 2005). Their interaction is disrupted either by mutations in RPGRIP1, found in patients with LCA, or by mutations in NPHP4, found in patients with SLSN (Roepman et al. 2005). 
The standard wire-haired dachshund (SWHD), the miniature long-haired dachshund (MLHD), and the pit bull terrier (PBT) are the only dog breeds to date known to be affected by crd (Kijas et al. 2004; Mellersh et al. 2006; Ropstad et al. 2007a). A mutation in canine RPGRIP1 has been demonstrated to cause autosomal recessive crd in MLHDs (Mellersh et al. 2006), while the genetic basis of the crd found in the PBT is still unknown (Kijas et al. 2004).

A family of SWHDs segregating recessively inherited crd was utilized to characterize the phenotype (Ropstad et al. 2007a,b) and, initially, to rule out candidate genes (Wiik et al. 2008). In this study, a whole-genome association of discordant sib-pairs genotyped with the canine single nucleotide polymorphism (SNP) array (Karlsson et al. 2007) was followed by fine-mapping and mutation detection to identify a deletion in NPHP4 that causes crd in these dogs. This mutation is the first nephroretinin mutation to cause retinal disease without kidney involvement.

\section{Results}

\section{Genome-wide association analysis}

A significant association was detected to chromosome 5 (CFA5) using sibTDT analysis of $~ 50,000$ SNPs in 13 cases and 13 controls selected as discordant sib-pairs from a pedigree of SWHD (Fig. 1). All samples were genotyped on the Affymetrix v2 canine SNP array. One control was excluded from further analysis based on low call rate $(<75 \%)$. On the basis of the close relationship of individuals within this pedigree, a Sibling Transmission Disequilibrium Test (sibTDT) analysis as part of the PLINK software (Purcell et al. 2007) was performed, and genome-wide significance $\left(P_{\text {rawtdT }}=4.8 \times 10^{-5}\right.$ and $\left.P_{\text {genometdT }}=0.00060\right)$ was achieved over the region CFA5: $61,122,335-67,576,960$. Homozygosity was detected in affected cases from 61.10 to $69.07 \mathrm{Mb}$ (Fig. 2A,B). Case-control association identified the same region but reported the highest association to a spurious SNP in the "shoulder" of the linkage region $\left(P_{\text {rawassoc }}=1.3 \times 10^{-5}, P_{\text {genomeassoc }}=\right.$ $5.2 \times 10^{-3}$; Fig. $\left.2 \mathrm{~B}\right)$. In this region, allelic frequencies differed maximally between cases and controls; however, one control dog was homozygous for the "affected" allele at the point of association (Chr5:69351798) and the foundation sire (201) was heterozygous in the near vicinity (although not at this SNP).

\section{Fine-mapping and mutation detection}

Fine-mapping and haplotype analysis of microsatellites of the region between $52.8 \mathrm{Mb}$ and $77.0 \mathrm{Mb}$, in 36 informative offspring, revealed crossovers at $61.4 \mathrm{Mb}$ and $72.0 \mathrm{Mb}$ (Fig. 1), thus it could not further reduce the area of association, despite including additional meiosis. The associated region contains $>70$ genes that were examined to find possible candidate genes expressed in the retina. DAB1 (55.3 Mb, outside region), AJAP1 $(62.0 \mathrm{Mb})$, VAMP3 $(64.4 \mathrm{Mb})$, and NPHP4 $(62.9 \mathrm{Mb})$ were identified as po-

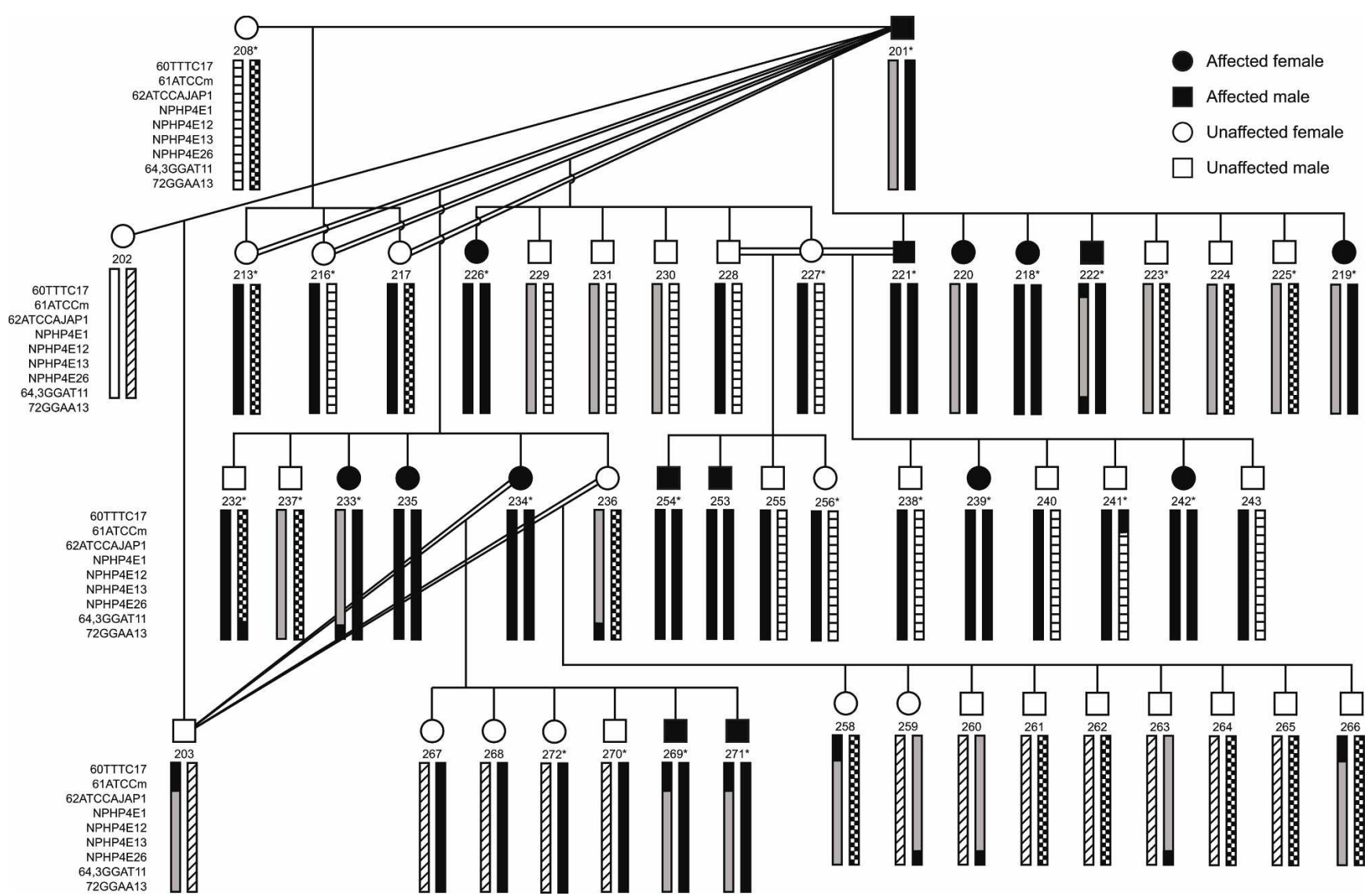

Figure 1. Pedigree of the dachshund family segregating crd that was used for genome-wide association. Discordant sibs of half sibs were genotyped $\left.{ }^{*}\right)$ on the canine SNP-array, and sibTDT genome-wide association was performed. Microsatellite markers in the associated CFA5 region were used for identification of recombinant breakpoints and haplotypes are shown for each dog. 

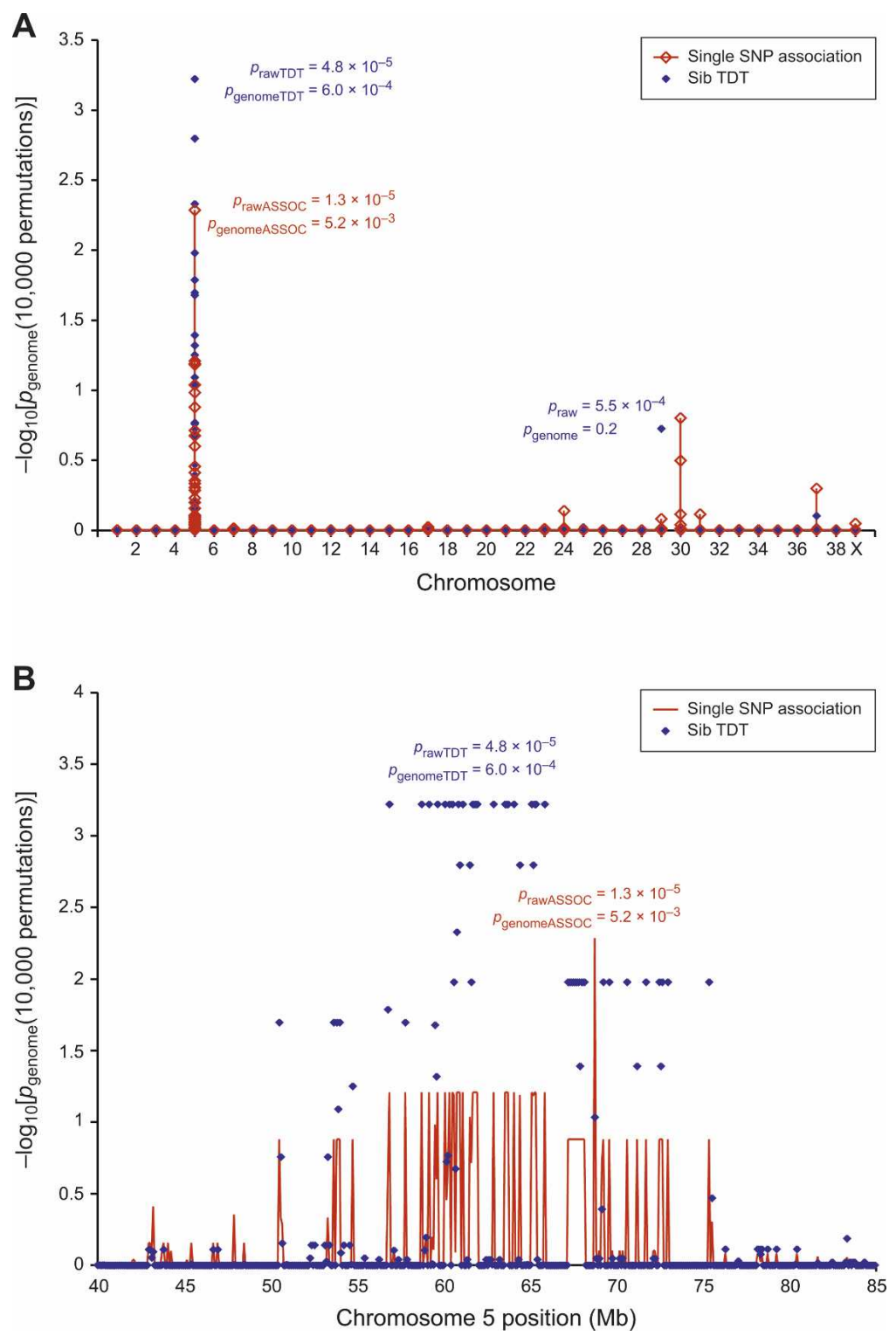

Figure 2. Genome-wide association mapping identified a locus on CFA5. (A) The best association was identified by sibTDT analysis $\left(P_{\text {genomeTDT }}=0.00060\right)$, but case-control association was also significant $\left(P_{\text {genomeassoc }}=5.2 \times 10^{-3}\right)$ using PLINK (Purcell et al. 2007). (B) Comparison of sibTDT and single SNP association statistics across the CFA5 region.

tential candidate genes. Exon sequencing, including exon-intron boundaries, showed no mutations in the first three genes. PCR products from exon 5 of NPHP4 showed a different size in unaffected (519 bp) and affected (339 bp) individuals and with both bands seen in carriers (Fig. 3A). Exon sequencing showed a deletion of $180 \mathrm{bp}$ in exon 5/intron 5 (location 62,913,591$62,913,770)$ in the affected animal, where only the first 18 bases of exon 5 were retained (Figs. 3C, 4). Screening of exon 5 in 84 unrelated SWHD revealed 8 carriers, giving a gene frequency of the mutated gene of $\sim 9.5 \%$ in the SWHD population. The exon screening also revealed five SNPs within exons, and 4 SNPs were found in intronic sequence close to exons (Supplemental Table S4). All of the expressed SNPs give rise to amino acid changes, but all are present as polymorphisms in multiple breeds and are not associated to disease within the dachshunds (Supplemental Table S4). No mutations were found in the exon/intron junctions. The putative mutation was not found in any of 32 examined dogs from eight different breeds (Supplemental Table S5).

\section{Transcript analysis}

Comparison of cDNA from affected and unaffected dogs using RT-PCR revealed complete exon-skipping of exon 5 in the affected animals (Fig. 3B,D). Translation of the 4819-bp cDNA sequence of the healthy dogs gives a 1429-amino-acid (aa) protein, while translation of the cDNA of the affected dog results in an early stop codon in exon 6 resulting in a short protein of 155 aa (Fig. 4). The RACE procedure of the 3 '- and $5^{\prime}$-ends gave a complete mRNA sequence, "EU659993," consisting of 4819 bp distributed on 30 exons. Exon 1 (73 bp) and the first $35 \mathrm{bp}$ of exon 2 are untranslated (5'-UTR), while the last $420 \mathrm{bp}$ of exon 30 are 3 '-UTR. The NPHP4 exons are located at the minus strand of CFA5 from $62,935,638$ to $62,819,750 \mathrm{bp}$. The amplification of the transcript ends revealed no differences between the affected and the unaffected dogs.

\section{Discussion}

Thirteen affected and 13 sex- and agematched controls were selected for genome-wide association and were genotyped using the Affymetrix version 2 SNP array. After association to CFA5, the haplotypes were used to identify a region of homozygosity among case individuals in the associated region. Both case-control association analysis and sibTDT identified the same general region of association to the disease trait with genome-wide significance. As has been previously demonstrated (Karlsson et al. 2007), a Mendelian recessive trait can be successfully mapped within a single dog breed with fewer than 15 cases and 15 control dogs. However, the SNP with the most significant case-control association probability was not in the region of the best sibTDT association. In this instance, where a relatively small number of individuals were analyzed and where these individuals were predominantly mismatched sibs, the employment of allelic case-control analysis was not the optimal analytical method. This study shows us that in such cases, supportive analyses, such as autozygosity mapping and TDT analysis, may be required to identify the best possible association. Since $6 \%$ of the genome is homozygous at the $0.5-\mathrm{Mb}$ level within a breed (Karlsson et al. 2007), simple homozygosity mapping will identify too many loci, and the power offered by information about trans- 
mission in a sibTDT test is valuable. The analysis demonstrates that lack of relationship among included individuals is not a prerequisite of successful association mapping and that knowledge of the pedigree structure can be used to better inform statistical results from the analysis. However, it is important to consider that the identified region will be considerably larger than for a case-control study where the typical associated region is 0.5-1 Mb in size (Karlsson et al. 2007; Salmon Hillbertz et al. 2007)

Resequencing of candidate genes within the region of homozygosity of affected dogs was conducted in parallel with finemapping. DAB1 coding for disabled homolog 1 (55.3 Mb), AJAP1adherens junction associated protein $(62.0 \mathrm{Mb})$, and VAMP3vesicle associated protein $(64.4 \mathrm{Mb})$ were excluded, but resequencing of the NPHP4 gene identified a 180-bp deletion in exon/intron 5 that causes exon skipping and early truncation of the protein in exon 6. This 155-aa, shortened form of NPHP4 contains the binding site for nephronophthisis 1 (NPHP 1 , also known as nephrocystin 1), which is known to interact with NPHP4 in kidney, but has lost the binding site for RPGRIP1, the protein known to interact with NPHP4 in retina. This is particularly interesting since mutations in NPHP4 have not yet been described as a cause of an eye disease without affecting the kidneys simultaneously. Mutations in RPGRIP1, however, have been reported to be a cause of LCA (Dryja et al. 2001; Gerber et al. 2001), while mutations causing residual RPGRIP1 activity may lead to phenotypes such as RP or crd, which are less severe than LCA (Cremers et al. 2002). Some RPGRIP1 gene mutations are also associated with recessive crd in human (Hameed et al. 2003) and dog (Mellersh et al. 2006).

More specifically, the RPGRIPinteracting domain of NPHP4 has previously been pinpointed to amino acids 591-960 of GenBank entry NP_055917, encoded by human exons 15-21 (Roepman et al. 2005), and is clearly not present in the 155-aa NPHP4 protein of affected dachshunds. The size of the deletion in NPHP4 and the colocalization and interaction with RPGRIP1 may explain why this mutation is causing pure crd and why this disease found in the SWHDs is relatively similar to the one found in MLHD, which has a RPGRIP1 mutation. Time of onset of the initial crd in the affected SWHDs varied from 10 mo to $3 \mathrm{yr}$ of age, with a complete retinal atrophy evident within $6 \mathrm{yr}$ (Ropstad et al. 2007a). The most characteristic opthalmoscopic changes were initial cellophane-like increase in tapetal sheen and marked pigment migration in the nontapetal fundus. The MLHD showed a granular appearance of the tapetal fundus at the age of 26 wk. Electrophysiologically, crd-affected SWHDs showed reduced cone-derived responses already at $5 \mathrm{wk}$, while the rodderived responses were less affected. At $52 \mathrm{wk}$, most affected animals still had recordable electroretinography (ERG) responses. In the MLHDs, the ERG responses were nonrecordable already at the age of $40 \mathrm{wk}$ (Mellersh et al. 2006).

The N-teminal region of NPHP4 is crucial for the interaction with NPHP1 (Mollet et al. 2005). Our findings show that the first 155 amino acids are intact in the mutated protein of the SWHD, and this may provide another explanation why the mutation does not affect the kidneys. The binding region with NPHP1 was known to be located in the first 176 amino acids of NPHP4 (Mollet et al. 2005), but this study refines the region further to the first 155 amino acids-assuming that NPHP4 has the same function
A

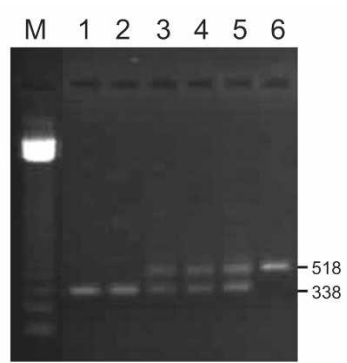

B

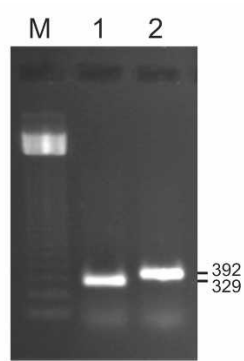

C

wt gegctctgtggtcttgctttgcagGTTGAGGCTGTATCACGGCACTCCCCGAGCCCTCCT

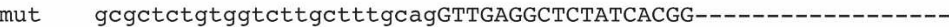
$* * * * * * * * * * * * * * * * * * * * * * * * * * * * * * * * * * * * * * * * * *$

wt GCACCCACTGCTCCAGGACCCTTTCGAACgtaagagacaaggcetggectggectgaggt mut

wt gttgctgtgaagtcctctcatcctccctatgactttcctggtgttatctgctttgactcc mut

wt atcttgttctcttctctgtgtaaaccattatcagcttaggtgatttatttaagggatta

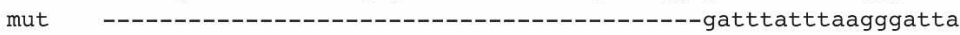
$* * * * * * * * * * * * * * * * * *$

wt ggacaaaagtgtcatgagcttggatggttgacgtaactgaaggaactgtaagcactagc mut ggacaaaagtgtcatgagcttggatggttgacgtaactgaaggaactgtaagcactagc $* * * * * * * * * * * * * * * * * * * * * * * * * * * * * * * * * * * * * * * * * * * * * * * * * * * * * * * * * * * *$

D wt CTCTCTCCAGGCTCTGTCATGTGGCTTTGGAATTCTTCGGATCTTTGGGAACAAACTGGA mut CTCTCTCCAGGCTCTGTCATGTGGCTTTGGAATTCTTCGGATCTTTGGCAACAAACTGGA $\begin{array}{llllllllllllllllllllllllllllll}S & L & Q & A & L & S & C & G & F & G & I & L & R & I & F & G & N & K & L & E\end{array}$

wt ATCTCCTACCTCTGCCTCCCAGGACAAGCGGTTGAGGCTGTATCACGGCACTCCCCGAGC $\begin{array}{llllllllllllllllllll}S & P & T & S & A & S & Q & D & K & R & \text { L } & R & L & Y & H & G & T & P & R & A\end{array}$

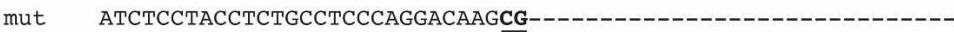
$\begin{array}{llllllllllllllllllll}S & P & T & S & A & S & Q & D & K & R\end{array}$

wt CCTCCTGCACCCACTGCTCCAGGACCCTTTCGAACAAAATAAACACATGACCCTCATTGA

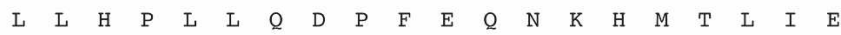
mut --$\mathrm{K}-$

wt GAGCTGCAGCCTGCAATACACTCTGAAGCCACACCCGCTCCTGGAGCCCGTCTTCCATCT

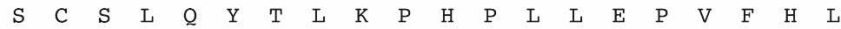
mut GAGCTGCAGCCTGCAATACACTCTGAAGCCACACCCGCTCCTGGAGCCCGTGTTCCATCT

Figure 3. A deletion in exon 5 is visualized on the genomic and transcript level. $(A)$ PCR products of exon 5 in affected dogs (339 bp, lanes 1,2), carrier dogs (heterozygotes, lanes 3-5), and unaffected dog (519 bp, lane 6). (B) PCR amplification of cDNA of one affected (329 bp, lane 1) and one healthy dog (392 bp, lane 2) using primers localized in exon 3 and exon 6. (C) Alignment of sequenced PCR product of mutant and wild-type DNA, where exonic sequence is represented in uppercase letters and intronic sequence in lowercase letters. $(D)$ cDNA. 


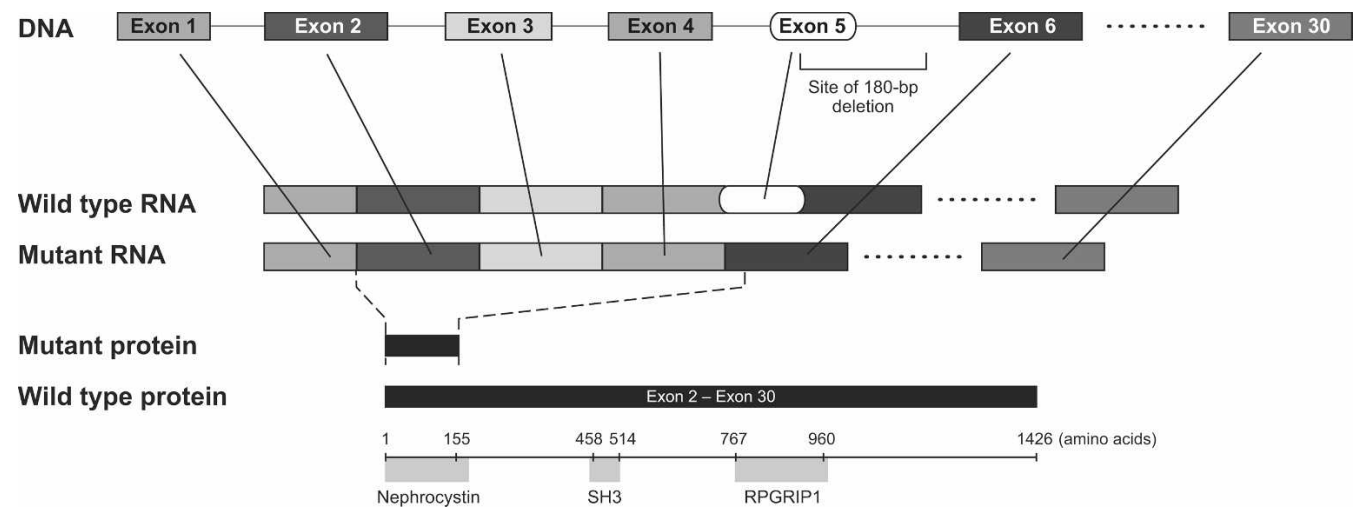

Figure 4. Illustration of the 180-bp deletion in exon 5 of the NPHP4 gene at DNA, RNA, and protein level. The mutation leads to exon-skipping of exon 5 and a stop codon in exon 6, which truncates the affected protein at 155 aa. The truncated protein contains the nephrocystin interacting domain but not the RPGRIP1 domain.

in humans and dogs-as no clinical or pathological symptoms of kidney disease were observed in the crd-affected dachshunds (oldest dog followed until $6 \mathrm{yr}$ of age). It will now be interesting to search for mutations in NPHP4 in human crd patients without kidney disease.

This paper demonstrates clearly that small canine pedigrees can be utilized for association mapping to identify mutations in genes of human relevance and that such data can be used to shed light on protein function as well as to generate new candidate genes for human disease.

\section{Methods}

\section{Animals and diagnosis}

Day blindness was diagnosed in a wire-haired standard dachshund, and a purpose-bred family of dogs confirmed that this most likely was a simple recessively inherited phenotype. Detailed clinical descriptions of the affected family have been reported previously (Ropstad et al. 2007a,b), establishing the disease as an early-onset crd. All procedures used in this study were in accordance with the Norwegian Animal Research Authority ("Forsøksdyrutvalget") and adhered to the Association for Research in Vision and Ophthalmology Statement for the Use of Animals in Opthalmic Vision and Research.

\section{Samples}

Blood samples were collected in EDTA-coated tubes from all the families segregating the disease and from two unaffected, unrelated dogs. Genomic DNA was isolated from the blood samples using DNeasy Tissue kit (Qiagen). Thirteen affected and 13 sexand age-matched controls (preferably full sibs) were selected for whole-genome SNP analysis (Fig. 1). As some of the dogs were euthanized, a number of samples from various tissues (including retina and muscle) were collected immediately post-mortem and frozen on liquid nitrogen for RNA analysis.

\section{Whole-genome association analysis}

From the SWHD family, 13 pairs of cases and controls were chosen. Where possible, mismatched full sibs $(n=10)$ were used in the analysis to equally distribute population stratification effects among cases and controls. Where a full sib-pair was not available, mismatched half-sibs were used ( $n=2$ pairs). The founding sire (case 201) and dam (control 208) were included in the analysis.
Genome-wide association analysis was undertaken using the Affymetrix version 2 Dog SNP Array "Platinum Panel" containing 49,663 SNP markers (Affymetrix). SNP genotypes were obtained by following the Affymetrix protocol for the human $500 \mathrm{~K}$ array protocol but with a smaller hybridization volume to allow for the smaller surface area of the canine array as described in Karlsson et al. (2007). Detailed information on the arrays is available at http://www.broad.mit.edu/mammals/dog/caninearray/.

The sibTDT was carried out on all 49,663 SNPs using the "Dfam" option in the analysis package PLINK (Purcell et al. 2007). Data were filtered to exclude individual calls with BRLMM calling probabilities lower than 0.01 , loci with low minor allele frequency $(<0.05)$, loci with low genotyping success rate (failed calls $>0.1$ ), and individual dogs with greater than $25 \%$ of genotypes missing. After applying these filters, 24,798 SNPs and 25 dogs (13 cases and 12 controls) remained in the analysis. The mean genotyping rate in the individual SWHDs included in the analysis was 96\%. Genome-wide significance was ascertained through permutation of phenotypes ( $n=10,000$ permutations) over all 24,798 SNPs in the analysis (EMP2 in PLINK).

Case-control association mapping using PLINK was applied to the same data with the same filtering parameters to test the efficacy of case-control association strategies in related samples. A region of association spanning from 57.6 to $72.8 \mathrm{Mb}$ was identified using the array analysis.

\section{Fine-mapping}

Primers for microsatellite analysis in the region of CFA5 (52.8 and $77.0 \mathrm{Mb}$ ) were identified by searching selected sequence windows of $\sim 200,000$ bp for microsatellite motifs. Tetra- and dinucleotide repeat microsatellites with more than 10 and 19 repeats, respectively, were selected. Primers for amplification of selected microsatellites were designed using Primer3 (http:// frodo.wi.mit.edu/cgi-bin/primer3/primer3_www.cgi) (Supplemental Table S1). One primer in each pair was labeled with fluorescein. For genotyping, PCR was carried out as described below with 28 cycles. The sizes of the alleles were estimated with an automated genotyper (ABI PRISM 3100 Genetic Analyzer, Applied Biosystems) with software for fragment analysis.

\section{Mutation screening}

Chromosomal locations of the predicted canine orthologs of the candidate genes were determined by using the http:// genome.ucsc.edu website. Primers were designed in introns, using primer3 (Supplemental Table S2), at a distance of $\sim 100-200$ 
bp from the intron/exon borders, to provide optimal PCR products for bidirectional sequencing. The primers for amplification on each of the canine NPHP4-exons were positioned in introns according to the described human sequence NM_015102 (Supplemental Table S3). The exon-specific primers for amplification of cDNA were based on the predicted canine sequence XM_546745.

PCR amplification reactions were performed using $83 \mu \mathrm{M}$ of each primer in a $15-\mu \mathrm{L}$ reaction containing $1.5 \mu \mathrm{L}$ of DNA prepared as described above, $1 \times$ Ammonium PCR buffer containing $1.5 \mathrm{mM} \mathrm{MgCl}_{2}, 83 \mu \mathrm{M}$ each of dATP, dCTP, dGTP, and dTTP, and 0.4 units Taq DNA polymerase (Ambion). After an initial denaturation at $95^{\circ} \mathrm{C}$ for $150 \mathrm{sec}$, samples were amplified for 34 cycles at $95^{\circ} \mathrm{C}$ for $30 \mathrm{sec}, 58^{\circ} \mathrm{C}$ for $40 \mathrm{sec}$, and $72^{\circ} \mathrm{C}$ for $50 \mathrm{sec}$, followed by a final extension of $72^{\circ} \mathrm{C}$ for $330 \mathrm{sec}$. All PCR products were bidirectionally sequenced using the PCR primers and the BigDye Terminator v3.1 Cycle Sequencing Kit (Applied Biosystems). Amplification was done in a $10-\mu \mathrm{L}$ reaction mixture containing $2 \mu \mathrm{L}$ of BigDye sequencing buffer, $1.5 \mu \mathrm{L}$ of BigDye sequencing enzyme, $1 \mu \mathrm{L}$ of PCR product, $0.5 \mu \mathrm{L}$ of sequencing primer $(5 \mathrm{pmol} /$ $\mu \mathrm{L}$ ), and $5 \mu \mathrm{L}$ of $\mathrm{dH}_{2} \mathrm{O}$. The sequencing reaction was performed by cycle sequencing with the following protocol: initially $95^{\circ} \mathrm{C}$ for $1 \mathrm{~min}$, then $28 \mathrm{cycles}$ each of $95^{\circ} \mathrm{C}$ for $10 \mathrm{sec}, 50^{\circ} \mathrm{C}$ for $15 \mathrm{sec}$, and $60^{\circ} \mathrm{C}$ for $2 \mathrm{~min}$. Sequence reaction products were purified by ammonium acetate precipitation and sequenced on the ABI3100.

\section{Transcript analysis}

One affected family member was compared with an unaffected English cocker spaniel (ECS) at the mRNA level. Total RNA was isolated from $30 \mathrm{mg}$ of muscle tissue using RNeasy fibrous tissue kit (Qiagen) following the manufacturer's instructions. The RNA was precipitated using standard procedures with ethanol, sodium acetate, and glycogen. The RNA was dissolved in $10 \mu \mathrm{L}$ of RNase free water and first-strand cDNA synthesis was performed using Superscript II RT following the manufacturer's descriptions. The final cDNA product was diluted 1:1 with RNase free water. Exonspecific primers were designed based on the predicted canine NPHP4 sequence XM_546745. PCR with these primers were performed as described above with $1 \mu \mathrm{L}$ of cDNA as template.

3 '- and 5'-RACE was performed to amplify the ends of the NPHP4 transcript in both dogs. RNA was isolated from $30 \mathrm{mg}$ of muscle tissue as described above, precipitated, and dissolved in RNase free water. mRNA for use in the Marathon RACE protocol was isolated from the total RNA using Dynabeads oligo-dT particles (Invitrogen). 5'- and 3'-end RACE was performed with GeneRacer Kit (Invitrogen) and Marathon cDNA Amplification Kit (Clontech), according to the manufacturers' protocols. PCR and nested PCR was performed using Kit-specific 5'- and 3'primers with Reverse end Forward gene-specific primers, and cloning of PCR products was performed using TOPO cloning kit. The inserts of the clones were amplified with M13 forward and reverse primer and sequenced with $\mathrm{T} 3$ and $\mathrm{T} 7$ primers.

\section{Acknowledgments}

We acknowledge the Norwegian School of Veterinary Science for funding the project and Casey Gates and the Broad Institute Genetic analysis platform for performing the genotyping. We thank Michael C. Zody for comments on the manuscript and Leslie Gaffney for practical help with the manuscript preparation.

\section{References}

Adams, N.A., Awadein, A., and Toma, H.S. 2007. The retinal ciliopathies. Ophthalmic Genet. 28: 113-125.
Allikmets, R., Shroyer, N.F., Singh, N., Seddon, J.M., Lewis, R.A., Bernstein, P.S., Peiffer, A., Zabriskie, N.A., Li, Y., Hutchinson, A., et al. 1997a. Mutation of the Stargardt disease gene (ABCR) in age-related macular degeneration. Science 277: 1805-1807.

Allikmets, R., Singh, N., Sun, H., Shroyer, N.F., Hutchinson, A. Chidambaram, A., Gerrard, B., Baird, L., Stauffer, D., Peiffer, A., et al. 1997b. A photoreceptor cell-specific ATP-binding transporter gene (ABCR) is mutated in recessive Stargardt macular dystrophy. Nat. Genet. 15: 236-246.

Arts, H.H., Doherty, D., van Beersum, S.E., Parisi, M.A., Letteboer, S.J., Gorden, N.T., Peters, T.A., Marker, T., Voesenek, K., Kartono, A., et al. 2007. Mutations in the gene encoding the basal body protein RPGRIP1L, a nephrocystin-4 interactor, cause Joubert syndrome. Nat. Genet. 39: 882-888.

Caridi, G., Murer, L., Bellantuono, R., Sorino, P., Caringella, D.A., Gusmano, R., and Ghiggeri, G.M. 1998. Renal-retinal syndromes: Association of retinal anomalies and recessive nephronophthisis in patients with homozygous deletion of the NPH1 locus. Am. J. Kidney Dis. 32: 1059-1062.

Cremers, F.P., van den Hurk, J.A., and den Hollander, A.I. 2002. Molecular genetics of Leber congenital amaurosis. Hum. Mol. Genet. 11: 1169-1176.

Danciger, M., Hendrickson, J., Lyon, J., Toomes, C., McHale, J.C., Fishman, G.A., Inglehearn, C.F., Jacobson, S.G., and Farber, D.B. 2001. CORD9, a new locus for arCRD: Mapping to 8p11, estimation of frequency, evaluation of a candidate gene. Invest. Ophthalmol. Vis. Sci. 42: 2458-2465.

Dryja, T.P., Adams, S.M., Grimsby, J.L., McGee, T.L., Hong, D.H., Li, T., Andreasson, S., and Berson, E.L. 2001. Null RPGRIP1 alleles in patients with Leber congenital amaurosis. Am. J. Hum. Genet. 68: $1295-1298$.

Gerber, S., Perrault, I., Hanein, S., Barbet, F., Ducroq, D., Ghazi, I. Martin-Coignard, D., Leowski, C., Homfray, T., Dufier, J.L., et al. 2001. Complete exon-intron structure of the RPGR-interacting protein (RPGRIP1) gene allows the identification of mutations underlying Leber congenital amaurosis. Eur. J. Hum. Genet. 9: $561-571$.

Hameed, A., Abid, A., Aziz, A., Ismail, M., Mehdi, S.Q., and Khaliq, S. 2003. Evidence of RPGRIP1 gene mutations associated with recessive cone-rod dystrophy. J. Med. Genet. 40: 616-619.

Hildebrandt, F., Jungers, P., Robino, C., and Grunfeld, J.-P. 2007 Nephronophthisis, medullary cystic kidney disease and medullary spong kidney disease. In Diseases of the kidney and urinary tract (ed. R. Schrier), pp. 478-501. Lippincott Williams \& Wilkins, Philadelphia, PA.

Ismail, M., Abid, A., Anwar, K., Qasim, M.S., and Khaliq, S. 2006. Refinement of the locus for autosomal recessive cone-rod dystrophy (CORD8) linked to chromosome 1q23-q24 in a Pakistani family and exclusion of candidate genes. J. Hum. Genet. 51: 827-831.

Karlsson, E.K., Baranowska, I., Wade, C.M., Salmon Hillbertz, N.H., Zody, M.C., Anderson, N., Biagi, T.M., Patterson, N., Pielberg, G.R., Kulbokas III, E.J., et al. 2007. Efficient mapping of mendelian traits in dogs through genome-wide association. Nat. Genet. 39: 1321-1328.

Khaliq, S., Hameed, A., Ismail, M., Anwar, K., Leroy, B.P., Mehdi, S.Q., Payne, A.M., and Bhattacharya, S.S. 2000. Novel locus for autosomal recessive cone-rod dystrophy CORD8 mapping to chromosome 1q12-Q24. Invest. Ophthalmol. Vis. Sci. 41: 3709-3712.

Kijas, J.W., Zangerl, B., Miller, B., Nelson, J., Kirkness, E.F., Aguirre, G.D., and Acland, G.M. 2004. Cloning of the canine ABCA4 gene and evaluation in canine cone-rod dystrophies and progressive retinal atrophies. Mol. Vis. 10: 223-232.

Mellersh, C.S., Boursnell, M.E., Pettitt, L., Ryder, E.J., Holmes, N.G., Grafham, D., Forman, O.P., Sampson, J., Barnett, K.C., Blanton, S. et al. 2006. Canine RPGRIP1 mutation establishes cone-rod dystrophy in miniature longhaired dachshunds as a homologue of human Leber congenital amaurosis. Genomics 88: 293-301.

Michaelides, M., Holder, G.E., Hunt, D.M., Fitzke, F.W., Bird, A.C., and Moore, A.T. 2005. A detailed study of the phenotype of an autosomal dominant cone-rod dystrophy (CORD7) associated with mutation in the gene for RIM1. Br. J. Ophthalmol. 89: 198-206.

Mollet, G., Silbermann, F., Delous, M., Salomon, R., Antignac, C., and Saunier, S. 2005. Characterization of the nephrocystin/nephrocystin-4 complex and subcellular localization of nephrocystin-4 to primary cilia and centrosomes. Hum. Mol. Genet. 14: $645-656$.

Olbrich, H., Fliegauf, M., Hoefele, J., Kispert, A., Otto, E., Volz, A., Wolf, M.T., Sasmaz, G., Trauer, U., Reinhardt, R., et al. 2003. Mutations in a novel gene, NPHP3, cause adolescent nephronophthisis, tapeto-retinal degeneration and hepatic fibrosis. Nat. Genet. 34: $455-459$. 
Cone-rod dystrophy in standard wire-haired dachshund

O’Toole, J.F., Otto, E.A., Hoefele, J., Helou, J., and Hildebrandt, F. 2007. Mutational analysis in 119 families with nephronophthisis. Pediatr. Nephrol. 22: 366-370.

Otto, E., Hoefele, J., Ruf, R., Mueller, A.M., Hiller, K.S., Wolf, M.T., Schuermann, M.J., Becker, A., Birkenhager, R., Sudbrak, R., et al. 2002. A gene mutated in nephronophthisis and retinitis pigmentosa encodes a novel protein, nephroretinin, conserved in evolution. Am. J. Hum. Genet. 71: 1161-1167.

Otto, E.A., Loeys, B., Khanna, H., Hellemans, J., Sudbrak, R., Fan, S., Muerb, U., O'Toole, J.F., Helou, J., Attanasio, M., et al. 2005 Nephrocystin-5, a ciliary IQ domain protein, is mutated in Senior-Loken syndrome and interacts with RPGR and calmodulin. Nat. Genet. 37: 282-288.

Parisi, M.A., Bennett, C.L., Eckert, M.L., Dobyns, W.B., Gleeson, J.G., Shaw, D.W., McDonald, R., Eddy, A., Chance, P.F., and Glass, I.A. 2004. The NPHP1 gene deletion associated with juvenile nephronophthisis is present in a subset of individuals with Joubert syndrome. Am. J. Hum. Genet. 75: 82-91.

Purcell, S., Neale, B., Todd-Brown, K., Thomas, L., Ferreira, M.A., Bender, D., Maller, J., Sklar, P., de Bakker, P.I., Daly, M.J., et al. 2007. PLINK: A tool set for whole-genome association and population-based linkage analyses. Am. J. Hum. Genet. 81: 559-575.

Roepman, R., Letteboer, S.J., Arts, H.H., van Beersum, S.E., Lu, X., Krieger, E., Ferreira, P.A., and Cremers, F.P. 2005. Interaction of nephrocystin-4 and RPGRIP1 is disrupted by nephronophthisis or Leber congenital amaurosis-associated mutations. Proc. Natl. Acad. Sci. 102: 18520-18525.

Ropstad, E.O., Bjerkas, E., and Narfstrom, K. 2007a. Electroretinographic findings in the Standard Wire Haired Dachshund with inherited early onset cone-rod dystrophy. Doc. Ophthalmol. 114: 27-36.
Ropstad, E.O., Bjerkas, E., and Narfstrom, K. 2007b. Clinical findings in early onset cone-rod dystrophy in the Standard Wire-haired Dachshund. Vet. Ophthalmol. 10: 69-75.

Salmon Hillbertz, N.H., Isaksson, M., Karlsson, E., Hellmen, E., Rosenberg Pielberg, G., Savolainen, P., Wade, C., von Euler, H. Gustafsson, U., Hedhammar, A., et al. 2007. A duplication of FGF3, FGF4, FGF9 and ORAOV1 causes dorsal hair ridge and predisposes to dermoid sinus in ridgeback dogs. Nat. Genet. 39: 1318-1320.

Sayer, J.A., Otto, E.A., O'Toole, J.F., Nurnberg, G., Kennedy, M.A., Becker, C., Hennies, H.C., Helou, J., Attanasio, M., Fausett, B.V., et al. 2006. The centrosomal protein nephrocystin- 6 is mutated in Joubert syndrome and activates transcription factor ATF4. Nat. Genet. 38: 674-681.

Udar, N., Yelchits, S., Chalukya, M., Yellore, V., Nusinowitz, S. Silva-Garcia, R., Vrabec, T., Hussles Maumenee, I., Donoso, L., and Small, K.W. 2003. Identification of GUCY2D gene mutations in CORD5 families and evidence of incomplete penetrance. Hum. Mutat. 21: 170-171.

Valente, E.M., Silhavy, J.L., Brancati, F., Barrano, G., Krishnaswami, S.R., Castori, M., Lancaster, M.A., Boltshauser, E., Boccone, L., Al-Gazali, L., et al. 2006. Mutations in CEP290, which encodes a centrosomal protein, cause pleiotropic forms of Joubert syndrome. Nat. Genet. 38: $623-625$.

Wiik, A.C., Ropstad, E.O., Bjerkas, E., and Lingaas, F. 2008. A study of candidate genes for day blindness in the standard wire haired dachshund. BMC. Vet. Res. 4: 23. doi: 10.1186/1746-6148-4-23.

Received February 6, 2008; accepted in revised form May 19, 2008. 


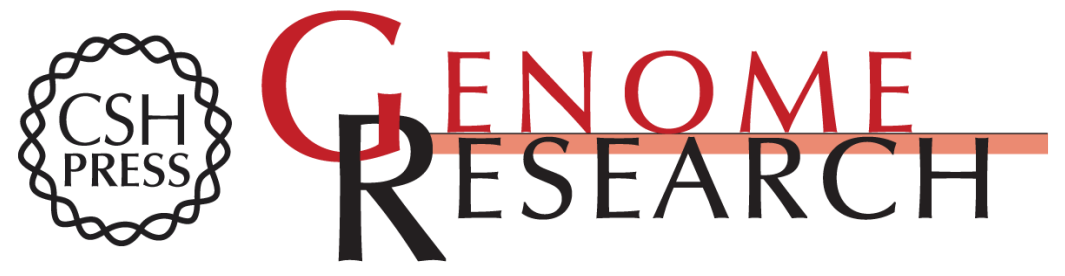

\section{A deletion in nephronophthisis 4 (NPHP4) is associated with recessive cone-rod dystrophy in standard wire-haired dachshund}

Anne Caroline Wiik, Claire Wade, Tara Biagi, et al.

Genome Res. 2008 18: 1415-1421 originally published online August 7, 2008

Access the most recent version at doi:10.1101/gr.074302.107

Supplemental Material

References

License

Email Alerting Service
http://genome.cshlp.org/content/suppl/2008/08/08/gr.074302.107.DC1

This article cites 31 articles, 6 of which can be accessed free at: http://genome.cshlp.org/content/18/9/1415.full.html\#ref-list-1

Receive free email alerts when new articles cite this article - sign up in the box at the top right corner of the article or click here.

\section{Affordable, Accurate Sequencing.}

